\section{Rodrigo Licéaga Reyes ${ }^{1}$ Manuel Arrascue Dulanto ${ }^{2}$ Mario Trejo Aguilar ${ }^{3}$ Adalberto Mosqueda Taylor ${ }^{4}$ Agustín Reyes Campos ${ }^{3}$}

'Docente de la especialidad Cirugía Oral y Maxilofacial, Hospital Juárez de México.

2Docente del Departamento de Medicina, Cirugía y Patología Oral. Facultad de Estomatología. Univerisdad Peruana Cayetano Heredia.

Univerisdad Peruana Cayetano Heredia.
${ }^{3}$ Cirujano Maxilofacial, Práctica Privada, México.

${ }^{3}$ Cirujano Maxilofacial, Práctica Privada, México.
${ }^{4}$ Docente del Departamento de Atención a la Salud. Universidad Autonoma de México-Xochimilco.

\section{Correspondencia}

Rodrigo Licéaga Reyes,

Tlaxcala 177-606 Col. Hipodomo Condesa, CP 06170 México DF, México.

Telef: 52-(55)-5286-9332 52-(55)-52869362.

E-mail: r_liceaga@ @otmail.com

Aceptado para publicación: 15 de junio del 2005

\title{
Quistes dentígeros múltiples en los maxilares, presentación de un caso
}

Licéaga R, Arrascue M, Trejo M, Mosqueda A, Reyes A. Quistes dentigeros múltiples en los maxilares, presentación de un caso. Rev Estomatol Herediana 2005;15(1): 73 - 76

\section{RESUMEN}

Presentamos un caso de un paciente de ocho años de edad con quistes dentigeros múltiples en los maxilares. Se discuten los posibles diagnósticos diferenciales y el tratamiento.

Palabras clave: QUISTE DENTÍGERO / ARCADA OSEODENTARIA MAXILAR

Multiple dentigerous cysts in jaws. Case study.

\section{ABSTRACT}

A case of an eight year-old child with multiple dentigerous cysts in jaw is presented. We discuss the possible differential diagnosis and its treatment.

Keywords: DENTIGEROUS CYST / JAW

\section{Introducción}

La naturaleza de las lesiones de los maxilares es muy variada y estas tienen características radiográficas que las hacen aparecer como imágenes radiolúcidas, mixtas y/o radiopacas. Estas imágenes pueden estar asociadas a manifestaciones clínicas como dolor, aumento de volumen y alteraciones en la sensibilidad, entre otras características.

Las lesiones de los maxilares en muchas ocasiones son originadas de las mismas estructuras embriológicas que forman los dientes, $\mathrm{y}$ por lo mismo pueden estar relacionadas con órganos dentarios en las distintas etapas de su desarrollo.

El quiste dentígero es considerado un quiste odontogénico del desarrollo (1), aunque se ha reportado también como de origen inflamatorio (2). Representa entre el $16,6 \%$ y $20 \%$ de los quistes de los maxilares $(3,4)$. En la población mexicana puede alcanzar hasta el $35 \%$ de los casos diagnosticados en servicios de patología bucal, con una ligera predilección por el sexo masculino $(5,6)$. Su incidencia esta en relación con la de los dientes permanentes retenidos, por lo que se asocia mas comúnmente a terceros molares inferiores, caninos superiores, premolares inferiores e incisivos superiores.

Clínicamente se caracteriza por provocar expansión en los maxilares, ser asintomático y ausencia clínica de uno o más dientes (7). Radiográficamente se describe típicamente como una zona radiolucida bien definida en relación con la corona de un diente retenido (8).

El comportamiento biológico del quiste dentígero es benigno, aunque se han informado casos aislados de transformación maligna de su epitelio (9).

Existen muy pocos reportes de quistes dentigeros múltiples (10-12), y generalmente se presentan solo en la mandíbula y no en ambos maxilares $(13,14)$. Solo existe un reporte de quistes en ambos maxilares en un paciente no sindrómico (15). El resto de los casos de los que se ha informado tienen un compromiso sistémico (16).

Presentamos el caso de un paciente con dos lesiones radiolúcidas independientes, una en maxilar y una en mandíbula, las cuales correspondieron histológicamente a quistes dentigeros.

\section{Presentación del caso}

Paciente masculino de 8 años de edad, que fue remitido al servicio de Cirugía Maxilofacial del Hospital Juárez de México por presentar aumento de volumen en el lado derecho del maxilar y el lado izquierdo de la mandíbula.

El interrogatorio no reveló antecedentes de relevancia para el padecimiento actual ni la existencia de enfermedades de carácter sistémico. Los padres del paciente refieren aumento de volumen lento y progresivo con 6 meses de evolución, el cual siempre fue asintomático. Hasta el momento no ha recibido ningún tratamiento.

A la exploración extraoral se encontró asimetría facial en ambos maxilares por expansión. El aumento de volumen 
era del lado derecho en el maxilar y del lado izquierdo en la mandíbula (Fig. 1).

A la palpación no se identificó aumento de temperatura local ni pulsación. La piel era normal. Se apreció ligera crepitación en ambas zonas afectadas. Intraoralmente se observó aumento de volumen en la zona maxilar derecha, alteraciones en el patrón de erupción con persistencia de dientes temporales incisivos superiores derechos (51 y 52) y ausencia clínica de los incisivos permanentes superiores derechos (11 y 12). La zona mandibular izquierda presentaba el aumento de volumen en relación con los molares temporales (74 y 75).

El resto de la cavidad bucal se encontró aparentemente sana, sin identificarse otras alteraciones.

Se tomaron varias radiografías simples donde se encontraron dos lesiones radiolúcidas, una en maxilar del lado derecho y otra en mandíbula izquierda. Ambas lesiones estaban en intima relación con órganos dentarios retenidos, aunque no existía una relación evidente entre ambas lesiones. En el estudio tomográfico se delimitaron las dimensiones de ambas lesiones.

Con fines diagnósticos se realizo una punción aspiradora en ambas lesiones obteniendo fácilmente líquido citrino claro en ambos casos. El líquido no fue enviado a análisis.

Los estudios de imagen evidenciaron dos lesiones independientes, una en maxilar y una en mandíbula, ambas completamente radiolúcidas con la corona de dientes retenidos íntimamente relacionados a cada una, así como otros dientes desplazados por las mismas lesiones (Fig. 2). Se observó la expansión de corticales, principalmente de la vestibular. El estudio tomográfico delimitó las lesiones, las cuales no reforzaron a la administración de contraste.

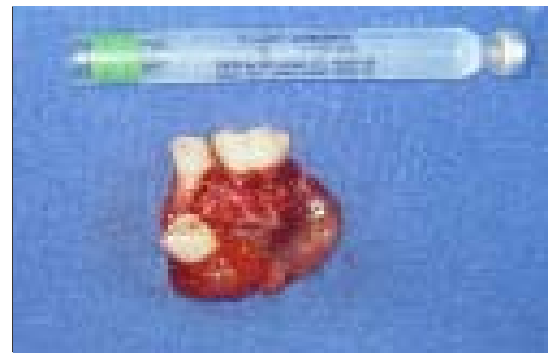

Figura 5. Aspecto macroscópico de quiste mandibular.
Con los hallazgos descritos se planteó el diagnóstico presuntivo de quistes dentígeros.

El tratamiento consistió en realizar bajo anestesia general la enucleación de ambas lesiones (17). La lesión maxilar se enucleo incluyendo los dientes $12 \mathrm{Y}$ 13. La lesión mandibular se enucleo incluyendo al diente 34 y conservando los dientes 33 y 35 (Fig. 3). Se utilizó sutura reabsorbible en ambos maxilares. Las dos muestras fueron enviadas a estudio histopatológico. Un día después fue dado de alta y se continuó su manejo en consulta externa (Fig. 4).

El estudio macroscópico evidenció dos especimenes, el primero asociado a la lesión maxilar y el segundo a la lesión mandibular. El primer espécimen fue de tejido blando con aspecto de saco quístico de forma ovoidal, superficie lisa de color blanco-grisáceo, el cual rodeaba a la corona de un diente incisivo permanente y se encontraba unido a su porción cervical. Midió 40x30x20 mm. Al corte fue de consistencia firme. El se-

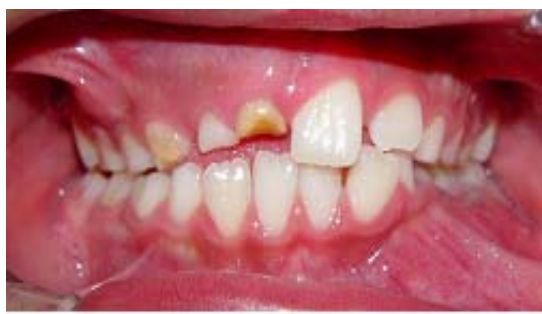

Figura 1. Vista intraoral inicial.

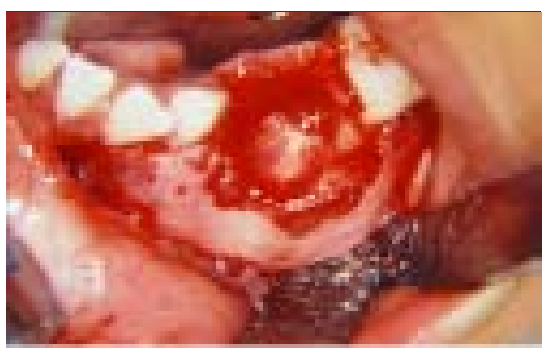

Figura 3. Vista operatoria de quiste mandibular.

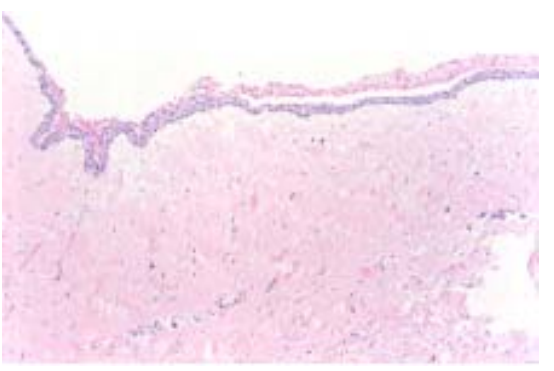

Figura 6. Pared de quiste dentígero con revestimiento de epitelio cuboidal delgado. gundo fue de tejido blando irregular, con aspecto de saco quístico abierto, el cual se encontró adherido al cuello de un premolar y rodeaba su corona y adicionalmente presentó adheridos a través de sus raíces dos dientes primarios. Su superficie y el revestimiento eran lisos, de color blanco-grisáceo y midió 35x25x20 mm(Fig. 5).

Al corte también fue de consistencia firme. Se incluyeron muestras representativas de ambos especimenes.

En las secciones microscópicas estudiadas se identificaron dos lesiones de naturaleza quística de aspecto similar, formadas por una gruesa cápsula de tejido fibroso laxo, el cual presenta abundante infiltrado inflamatorio de tipo mixto y predominio linfoplasmocitario. La superficie se observó revestida por epitelio escamoso estratificado no queratinizado, la cual mostró zonas de acantosis irregular y espongiosis, así como zonas de epitelio cuboidal delgado, compatible con el epitelio reducido del órgano del esmalte (Fig. 6).

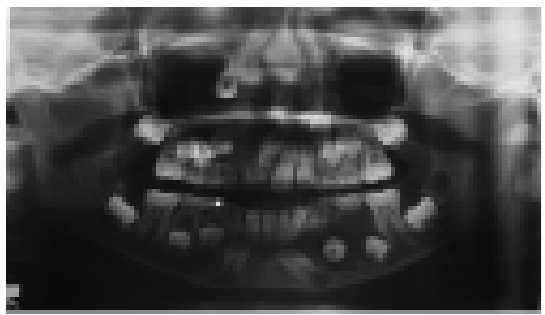

Figura 2. Radiografía panorámica inicial.

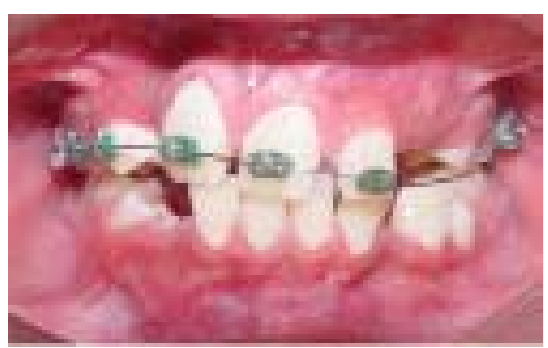

Figura 4. Vista intraoral actual.

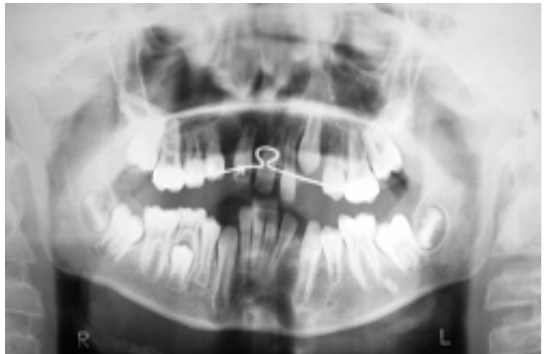

Figura 7. Radiografía panorámica actual. 
El diagnóstico final fue de quistes dentígeros (dos) con proceso inflamatorio crónico inespecífico asociado.

A los tres meses de la cirugía fue remitido a ortodoncia donde actualmente se encuentra bajo tratamiento (Fig. 7).

\section{Discusión}

Diferentes padecimientos ocasionan lesiones radiolucidas en los maxilares que provocan perdida ósea, mal posición dentaria y que requieren en la mayoría de las ocasiones de tratamiento quirúrgico. Es indispensable basarse en los hallazgos clínicos para descartar diferentes lesiones así como para poder decidir en que casos se requieren de estudios especiales que nos permitan relacionarlo con el diagnóstico clínico y corroborarlo por medio de la histopatología.

Pocos padecimientos ocasionan lesiones radiolúcidas de manera simultánea en ambos maxilares y sobre todo cuando no existe alguna alteración genética (18) o un padecimiento sistémico. Los quistes dentigeros son en la gran mayoría de las ocasiones lesiones solitarias, $\mathrm{y}$ al igual que en este caso no se asocian con enfermedades sistémicas excepto aquellos padecimientos que cursan con un mayor número de retenciones dentarias.

El diagnóstico diferencial de las lesiones de los maxilares debe basarse en los antecedentes, el examen clínico y los hallazgos radiográficos. En las lesiones radiolúcidas deben incluirse diferentes tumores y quistes odontogénicos, lesiones fibro-óseas en estados inmaduros, lesiones de células gigantes (19), así como otro tipo de tumores tanto benignos comos malignos.

Una de la herramientas diagnósticas más útiles en las lesiones radiolúcidas es la punción aspiradora, la cual permite determinar la naturaleza sólida o quística de la lesión. Incluso la calidad del contenido y su extendido para estudio citológico permite en ocasiones determinar la naturaleza de la lesión.

Pocas son las lesiones radiolúcidas que se presentan de manera múltiple en los maxilares. Entre los posibles diagnósticos diferenciales podemos mencionar al síndrome de Gorlin-Goltz, a la mucopolisacaridosis, al hiperparatiroidismo, al querubismo y ocasionalmene a los hemangiomas y las malformacio- nes vasculares, así como lesiones malignas como la histiocitosis de células de Langerhans y el mieloma múltiple.

La variabilidad del síndrome de Gorlin-Goltz es muy amplia y los pacientes pueden ser fenotipicamente imperceptibles hasta presentar la mayoría de las características clínicas que se presentan en el síndrome, el cual se caracteriza por presentar queratoquistes odontogénicos múltiples en los maxilares, los que se presentan en promedio en número de seis, y en un rango de edad entre la primera y la séptima década de la vida (20). Los quistes mandibulares son tres veces más frecuentes que los quistes maxilares (21). Estos quistes pueden ser sumamente grandes pero en raras ocasiones causan síntomas. Provocan desplazamiento dental y en ocasiones reabsorción radicular, pero muy rara vez fractura patológica $(22,23)$. Rara vez perforan la corteza e invaden tejidos blandos. En el caso que se reporta se descarto esta posibilidad diagnostica debido a que en la punzo aspiración se obtuvo un liquido citrino claro, hallazgo que difiere de lo observado en el queratoquiste odontogénico, del cual se obtiene un liquido blanquecino de aspecto lechoso que corresponde a la queratina.

Otra probabilidad diagnóstica es la mucopolisacaridosis. Esta es una familia de enfermedades metabólicas que resultan de la deficiencia de enzimas lisosomicas involucradas en la degradación de glucosaminoglicanos. Las principales manifestaciones orales son labios alargados, protusión de la lengua, dientes ampliamente espaciados, que exhiben atrición severa. Los incisivos pueden tener cierto grado de corona cónica pero son de otra forma estructuralmente normales. La presencia de macroglosia puede ocasionar mordida abierta anterior. Generalmente la erupción dental esta retrasada. Los segundos molares primarios o primeros y segundos molares permanentes están frecuentemente en posición distoangular $(24,25)$. Comúnmente se presentan áreas de destrucción ósea que se han designado como quistes dentigeros. Están frecuentemente presentes a los tres años de edad y más comúnmente involucran a los segundos molares primarios y primeros y segundos molares permanentes mandibulares. El margen de la radiolucidez es suave y claramente definido (26).

Estas lesiones representan acumulación de dermatan sulfato en folículos dentales hiperplasicos, y ocurren en muchas de las variedades de presentación de la mucopólisacaridosis (27). El tratamiento de estas lesiones pericoronales es similar al de quistes dentigeros convencionales (enucleación con el diente afectado).

Otra posibilidad diagnostica fue el hiperparatiroidismo, el cual es una alteración generalizada del metabolismo del calcio, del fósforo y del hueso que resulta del aumento en la secreción de la hormona paratiroidea. Esta concentración excesiva de la hormona circulante causa hipercalcemia e hipofosfatemia.

La mayoría de los pacientes tienen otros síntomas antes de presentar lesiones esqueléticas radiográficamente evidentes. La gradual disminución de calcio en los huesos produce una descalcificación generalizada y zonas de rarefacción de aspecto quístico localizado, que reciben el nombre de osteítis fibrosa quistica. Los huesos comúnmente afectados son los huesos largos, vértebras, pelvis, cráneo, mandíbula y huesos del carpo y del tarso.

Las lesiones de aspecto quístico se observan en menos del 10\% de los casos. Histológicamente son similares a los granulomas centrales y periféricos de células gigantes. Cuando los maxilares están afectados, los dientes se tornan móviles, aunque conservan su vitalidad (28).

En nuestro caso los niveles séricos de calcio, fósforo, fosfatasa alcalina y fosfatasa ácida resultaron normales. A diferencia de las lesiones del hiperparatiroidismo, en nuestro caso las imágenes están en íntima relación con las coronas de dientes retenidos, lo cual es una característica inusual en esta endocrinopatía.

Otras posibilidades diagnosticas fueron el querubismo y los hemangiomas o malformaciones vasculares intraoseas, pero estas lesiones al igual que el hiperpartiroidismo no se relacionan a las coronas de piezas dentarias retenidas. Por otro lado al realizar la punción aspiración en el caso que se reporta se obtuvo un liquido citrino claro y si las lesiones reportadas hubieran sido de tipo vascular se hubiera obtenido sangre y no un líquido citrino claro. 
La histiocitosis de células de Langerhans es una enfermedad que rara vez se encuentra en la cavidad oral y que puede presentarse en cualquier grupo de edad Se incluye como diagnostico diferencial porque produce lesiones osteolíticas usualmente múltiples cuando se manifiesta de manera agresiva (29).

El mieloma múltiple puede ser considerado principalmente por la imagen radiográfica descrita típicamente como zonas radiolúcidas múltiples en “sacabocado". Nuestro caso no corresponde al grupo edad del esta neoplasia, la que se presenta mas comúnmente en pacientes de mayor edad y a menudo con sintomatología sistémica asociada a la neoplasia (30).

\section{Conclusiones}

Aunque el tratamiento de las lesiones quísticas de los maxilares puede ser aparentemente simple, es muy importante siempre considerar todas las opciones diagnósticas, especialmente cuando se considera la posibilidad de un padecimiento sistémico.

Las lesiones quisticas múltiples de los maxilares no son frecuentes, mucho menos en ambos maxilares como lo fue este caso.

\section{Agradecimientos}

Al Dr. Edgar Torres Ortiz y a la Dra. Montserrat Camón Villanueva de la Universidad Tecnológica de México (UNITEC) del Departamento de Ortodoncia de Posgrado.

\section{Referencias bibliograficas}

1. Kramer IRH, Pindborg JJ, Shear M: Histological Typing of Odontogenic Tumors. (International Histologic Clasification of Tumors, ed. 2.) Berlin: Springer-Verlag, 1992, p 1-42

2. Benn A, Altini M: Dentigerous cyst of inflamatory origin. A clinicopathologic study. Oral Surg Oral Med Oral Pathol Oral Radiol Endod 1996;81:203-9.

3. Shear M. Cysts of the oral regions. Oxford: Wright. 1992.

4. Bodner L: Cyst lesions of the jaws in children, Int $\mathrm{J}$ Pedriatr Otorhinolaryngol 2002;62: 25-9.

5. Ledesma-Montes C, HernandezGuerrero JC, Garces-Ortiz M.: ClinicoPathologic Study of Odontogenic Cysts in a Mexican Sample Popula- tion. Arch Med Res 2000; 31: 373-6.

6. Mosqueda Taylor A, Irigoyen Camacho ME, Díaz Franco MA, Torres Tejero MA. Quistes odontogénicos. Análisis de 856 casos. Med Oral 2002;7:89-96.

7. Neville B, Damm D, Allen C, Bouquot J. Oral \& Maxillofacial Pathology. Philadelphia: W.B.Sauders Company. 2002. p 590-3.

8. Tsukamoto G, Sasaki A, Akiyama T, Ishikawa T, Kishimoto K, Nishiyama A, et al: A radiologic analysis of dentigerous cyst and odontogénic keratocysts associated with mandibular third molar. Oral Surg Oral Med Oral Pathol Oral Radiol Endod 2001; 91:743-7.

9. Yasuoka T, Yonemoto K, Kato Y, Tatematsu N. Squamous cell carcinoma arising in a dentigerous cyst. J Oral Maxillofac Surg 2000;58:900-5.

10. Ustuner E, Fitoz S, Atasoy C, Erden I, Akyar S: Bilateral maxillary dentigerous cyst: A case report. Oral Surg Oral Med Oral Pathol Oral Radiol Endod 2003; 95:632-5.

11. O’Neil DW, Mosby EL, Lowe JW. Bilateral mandibular dentigerous cysts in a five-year-old child: report of a case. ASDC J Dent Child 1989;56:382-4.

12. Sands T, Tocchio C. Multiple dentigerous cysts in a child. Oral Health 1998;88:27-29.

13. Ko KS, Dover DG, Jordan RC. Bilateral dentigerous cysts-report of an unusual case and review of the literature. J Can Dent Assoc 1999; 65:49-51.

14. Crinzi RA. Bilateral dentigerous cysts of the mandible. Oral Surg Oral Med Oral Pathol 1982;54:367.

15. Norris L, Piccoli P, Papageorge MB. Multiple dentigerous cysts of the maxilla and the mandible: report of a case. J Oral Maxillofac Surg 1987;45:694-7.

16. Colavita $\mathrm{N}$ et al: Calvarial doughnut lesions with osteoporosis, multiple fractures, dentinogenesis imperfecta and tumours changes in the jaws. Australas Radiol 1984; 28:226-31.

17. Albert B: Surgical technique in management of benign cyst and tumors of the jaws. Oral Maxillofac Surg Clin NAm 1991; 3:5-20.

18. Anderson DE, Cook WA: Jaws cyst and the basal cell nevus syndrome,
J Oral Surg 1966; 24:15-26.

19. Bruce KW, Bruwer A, Kennedy RL: Familiar intraosseus fibrous swelling of the jaws ("cherubism”). J Oral Surg 1953; 6:995-1014.

20. Dominguez FR, Keszler A: A Comparative study of keratocysts associated and non-associated with nevoid basal cell carcinoma syndrome. J Oral Pathol 1988; 17: 39-42.

21. Woolgar JA, Rippin JW, Browne RM: The odontogenic keratocyst and its occurrence in the nevoid basall cell carcinoma syndrome. Oral Surg Oral Med Oral Pathol. 1987;64:727-30.

22. Bramley P: The odontogenic keratocyst: An approach to treatment. Int J Oral Surg 1974; 3:337-41.

23. Browne RM: The odontogenic keratocyst: Histological features and their correlation with clinical behavior. Br Dent J 1971;131:249-59.

24. Downs AT, Crisp T, Ferretti G: Hunter's syndrome and oral manifestations: A review. Pediatr Dent. 1995; 17:98-100.

25. Gardner DG: The oral manifestation of Hurler's syndrome. Oral Surg Oral Med Oral Pathol 1971;32:46-57.

26. Worth HM: Hurler's syndrome: A study fo radiologic apparencces in the jaws. Oral Surg Oral Med Oral Pathol 1966;22:21-35.

27. Roberts MW, Barton NW, Constantopoulos G, Butler DP, Donahue AH. Occurrence of multiple dentigerous cysts in a patient with the Morateax-Lamy syndrome (mucopolysaccharidosis, type VI). Oral Surg Oral Med Oral Pathol 1984;58:169-75.

28. Silverman S Jr, Ware WH, Gillooly C: Dental aspects of hiperparathyroidism. Oral Surg Oral Med Oral Pathol 1968; 26:184-9.

29. Milian MA, Bagan JV, Jimenez Y, Pérez A, Scully C. Langerhans' Cell Histiocitosis restricted to the oral mucosa. Oral Surg Oral Med Oral Pathol Oral Radiol Endod. 2001;91:76-9

30. Pissano JJ, Coupland R, Chen SY, Miller AS. Plasmacytoma of the oral cavity and jaws: a clinicopathologic study of 13 cases. Oral Surg Oral Med Oral Pathol Oral Radiol Endod. 1997;83:265-71. 